\title{
Sensitivity of Blood Volume Monitoring for Fluid Status Assessment in Hemodialysis Patients
}

\author{
Francisco Maduell Marta Arias Elisabet Massó Néstor Fontseré \\ Montserrat Carrera Manel Vera Aleix Cases Josep M. Campistol \\ Department of Nephrology, Hospital Clinic Barcelona, Barcelona, Spain
}

\section{Key Words}

Bioimpedance · Blood volume monitoring · Fluid overload .

Fluid status assessment $\cdot$ Hemodialysis

\begin{abstract}
Background/Aims: This study investigates the use of blood volume monitoring (BVM) markers for the assessment of fluid status. Methods: Predialysis fluid overload (FO) and BVM data were collected in 55 chronic hemodialysis patients in 317 treatments. Predialysis FO was measured using bioimpedance spectroscopy. The slope of the intravascular volume decrease over time normalized by ultrafiltration rate (Slope4h) was used as the primary BVM marker and compared against FO. Results: Average relative blood volume curves were well separated in different FO groups between 0 and 5 liters. Receiver-operating characteristics analysis revealed that the sensitivity of BVM was moderate in median FO ranges between 1 and 3 liters (AUC 0.60$0.65)$, slightly higher for volume depletion of FO $<1$ liter (AUC 0.7) and highest for excess fluid of FO $>3$ liters (AUC 0.85 ). Conclusion: Devices that monitor blood volume are well suited to detect high FO, but are not as sensitive at moderate or low levels of fluid status.
\end{abstract}

Copyright $\odot 2013$ S. Karger AG, Basel

\section{Introduction}

Despite many technological advances in hemodialysis therapy, the management and optimization of fluid status remains a major challenge in the field of renal replacement therapy [1]. Fluid overload (FO)-related hospitalizations are high [2] and it has been shown that patients who present long-term FO suffer from increased mortality [3]. A multicenter study in eight European dialysis centers has revealed that $25 \%$ of all patients are volume overloaded [4].

Fluid management comprises two main aspects: (i) the short-term (intradialytic) preservation of blood volume to avoid hypotensive episodes, and (ii) the long-term maintenance of fluid status below a critical level beyond which cardiovascular damage may occur.

Usually, patients with high FO tend to be more stable during the treatment because of increased vascular refilling $[5,6]$. Some patients, however, may present high FO but still suffer from intradialytic episodes, possibly due to cardiac impairment, antihypertensive medication or other factors degrading vascular refilling [7]. It has been proposed that careful long-term fluid reduction in these patients may improve their cardiac status and subsequently lead to more intradialytic stability [4].

\section{KARGER}

E-Mail karger@karger.com

www.karger.com/bpu
(C) 2013 S. Karger AG, Basel

0253-5068/13/0353-0202\$38.00/0
Francisco Maduell Canals, MD

Servicio de Nefrología, Hospital Clinic Barcelona

C/Villarroel, 170

ES-08036 Barcelona (Spain)

E-Mail fmaduell@clinic.ub.es 
Blood or plasma volume monitoring was originally introduced as a tool to deal with the first aspect, i.e. to reduce intradialytic morbid events by controlling the ultrafiltration rate (UFR). Recently however, its utility for fluid status assessment has come more into focus [8-11]. Some authors have proposed to categorize patients' fluid status by the shape of their intradialytic blood volume reduction or postdialytic volume rebound curves [12-15]. Others have developed quantitative markers based on blood volume monitoring (BVM) to assess fluid status $[11,16]$.

Even though relationships with other clinical signs linked to fluid status like blood pressure (BP) or inferior caval vein diameter were found, concerns were raised about the high intraindividual variations $[17,18]$. Moreover, it has been reported that BVM markers allowed detecting excess fluid but not depletion [16], suggesting that BVM markers may not have the same sensitivity over a wide range of FO. Finally, calibration of the markers against an absolute level of FO measured in liters is still lacking.

It is the aim of this work to investigate the aforementioned points by using a quantitative measure of FO based on bioimpedance spectroscopy as a reference.

\section{Methods}

\section{Study Design}

Fifty-five clinically stable hemodialysis patients were selected in this observational cross-sectional study in March 2011 in the Hospital Clinic Barcelona (Spain). All patients underwent standard 4-5 h online HDF treatments on Fresenius 5008 machines. The UFR was kept constant within most treatments. Each patient was followed up over 7 consecutive treatments. BVM was performed during each of the seven treatments using a Fresenius blood volume monitor which provided relative blood volume (RBV) curves. FO was measured once in each patient. Pre- and postdialysis body weights were collected and systolic BP (SBP) and diastolic BP (DBP) were measured before and after dialysis. All data (including dialysis machine data such as ultrafiltration volume (UFV)) were stored in a database (Therapy Monitor and NefroLink; Fresenius Medical Care, Germany) for later analysis. Intradialytic BVM data was stored at a rate of one sample per minute.

\section{Measurement of Fluid Overload}

FO was determined before cannulation using a bioimpedance spectroscopy device (BCM body composition monitor; Fresenius Medical Care). The device measures whole-body bioimpedance over a frequency range from $5 \mathrm{kHz}$ to $1 \mathrm{MHz}$, and determines extracellular and total body water resistance by means of Cole modeling [19]. Unlike single- or mono-frequency devices, the BCM determines extra- and intracellular water volumes independently of each other, and it calculates FO independently of body composition by using the Chamney model which takes into account differences in adipose and lean tissue hydration [20]. FO is defined in this context as excess extracellular water. The device has been validated against dilution reference methods [21], dual-energy x-ray absorptiometry and the Fuller 4C model [22] in healthy subjects, dialysis patients, liver cirrhosis and palliative cancer patients. The difference between pre- and postdialysis measured FO corresponded very well with the removed UFV (mean paired difference 0.02 liter); a complete validation overview has been published elsewhere [23].

None of the patients had been measured with the BCM before, and the clinical staff was blinded to the BCM results in order not to influence UFV. FO was measured once in each patient. In order to increase the number of $\mathrm{FO}$ values, $\mathrm{FO}$ was additionally estimated on the three consecutive treatments before and after the BCM measurement day, using differences in preweight according to the following equation:

$$
\begin{aligned}
\mathrm{FO}_{\text {estimation }}= & \mathrm{FO}_{\mathrm{BCM} \_ \text {day }}+\text { preweight }_{\text {estimation_day }}- \\
& \text { preweight }_{\mathrm{BCM} \_ \text {day }}
\end{aligned}
$$

This approach assumes that treatment-to-treatment changes in preweight over no more than a few days reflect mainly changes in FO, and that body composition remains stable within this short time period.

\section{Definition of Volume Markers}

An overview of the different volume markers is provided in table 1. Three markers based on BVM were used: (1) the Slope $4 \mathrm{~h}$ which was defined as the linear slope of the RBV decrease over the whole treatment, normalized by UFR [\%/h/(l/h)]; (2) RBV in [\%] that was reached at the end of the treatment, and (3) volume index, defined as the RBV slope over the full treatment and normalized by UFR over postweight $(\triangle \mathrm{RBV} / \mathrm{h} /(\mathrm{UFR} /$ postweight $))$ as proposed by Agarwal et al. [16].

\section{Data Analysis}

Slopes were determined by means of robust iterative weighted least squares regression as provided by the statistical software in order to attenuate the effect of outliers on the results. Receiveroperating characteristic (ROC) curves were used to assess the discrimination abilities of a marker. They depict the true-positive rate over the false-negative rate. A ROC curve on the identity line indicates a completely random assignment, while a curve that quickly rises to $>90 \%$ and stays there for increasing false-negative rates indicates very good classification ability. ROC curves for the volume markers were determined for different cutoff values of FO, including area under the ROC curve (AUC) as a measure of discriminatory power and their $95 \%$ confidence intervals (CI). AUC confidence intervals were determined by bootstrapping, using 5,000 iterations for each AUC value. All analyses were performed using Matlab R2011a.

\section{Results}

Patient characteristics are listed in table 2. Each of the 55 patients was measured over a 7 -treatment observation period, giving a total number of $55 \times 7=385$ treatments. BVM readings were available in 317 of 385 treatments. 
Table 1. Overview of different volume markers and their correlations with fluid overload (FO)

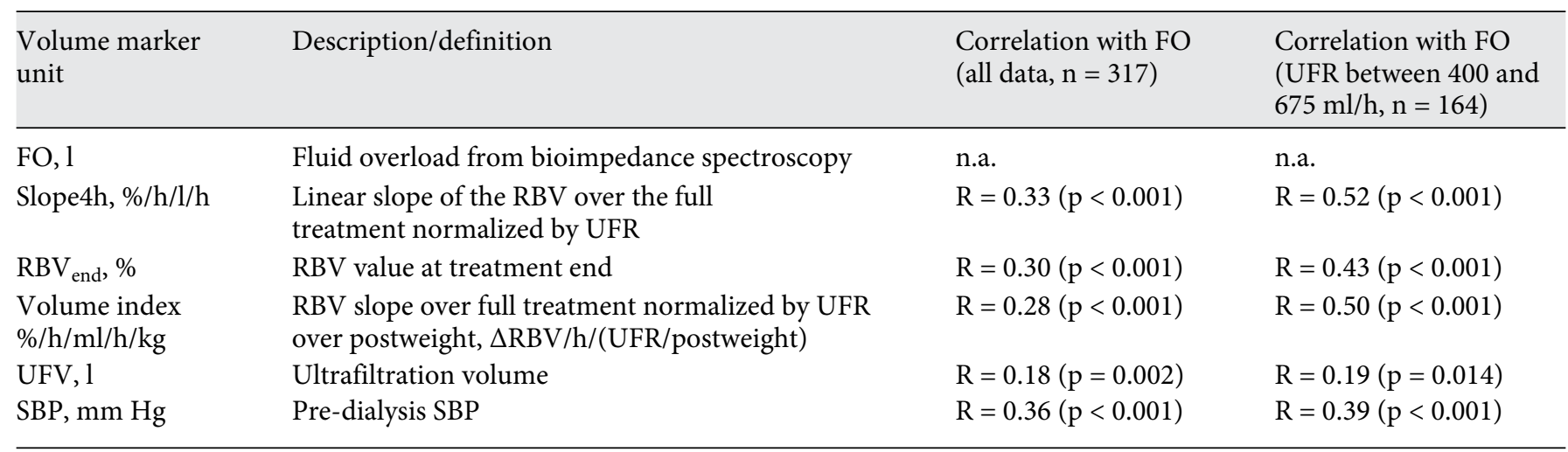

Table 2. Patient characteristics ( $\mathrm{n}=55$ patients, 317 treatments)

\begin{tabular}{lc}
\hline Characteristics & Mean \pm SD \\
\hline Age, years & $63 \pm 13$ \\
Dialysis vintage, months & $76 \pm 93$ \\
Males/females & $37 / 18$ \\
Height, cm & $166 \pm 10$ \\
Diabetes mellitus, patients, $\mathrm{n}(\%)$ & $15(27)$ \\
Weight before HD, kg & $71.9 \pm 15.0$ \\
Weight after HD, kg & $69.5 \pm 14.8$ \\
BMI before dialysis, kg/m ${ }^{2}$ & $26.0 \pm 3.6$ \\
UF volume, l & $2.77 \pm 0.98$ \\
UF rate, ml/h & $568 \pm 205$ \\
Treatment duration, min & $295 \pm 35$ \\
SBP before HD, mm Hg & $132 \pm 28$ \\
DBP before HD, mm Hg & $63 \pm 17$ \\
SBP after HD, mm Hg & $129 \pm 26$ \\
DBP after HD, mm Hg & $61 \pm 17$ \\
Fluid overload before HD, l & $2.3 \pm 1.9$ \\
Fluid overload after HD, l & $-0.5 \pm 2.0$ \\
Extracellular volume, l & $16.2 \pm 3.5$ \\
Intracellular volume, l & $16.1 \pm 3.4$ \\
Antihypertensive medications, n & $0.87 \pm 1.04$ \\
Albumin, g/l & $37.3 \pm 3.8$ \\
Hb, g/dl & $10.7 \pm 1.3$ \\
\hline
\end{tabular}

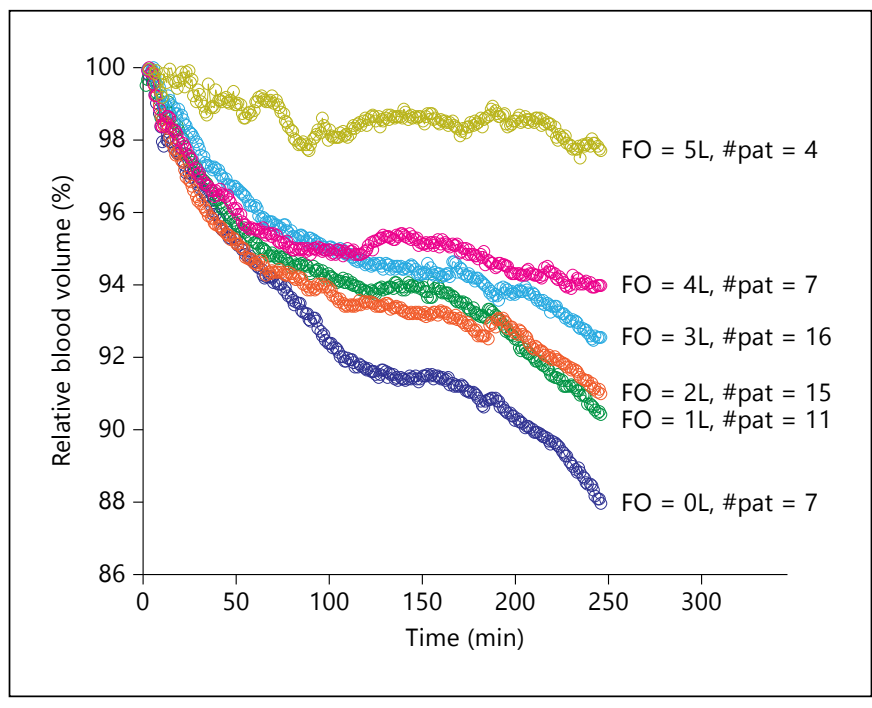

Fig. 1. RBV curves at different levels of predialysis FO. Only treatments with a UFR between the 25 th and 75 th percentile (400-675 $\mathrm{ml} / \mathrm{h}$ ) and treatment time $>10$ th percentile $(>245 \mathrm{~min})$ were included. The RBV curves were cut at 245 min to ensure that every minute had the same number of averages. In 20 of these 146 measurements, UFR was not constant (either UFR control on, or change during treatment).

75 th percentile, corresponding to $400-675 \mathrm{ml} / \mathrm{h}, \mathrm{n}=164$ ), in order to become independent of the effects of extreme UFRs on blood volume.

\section{RBV Curves Grouped by Fluid Overload}

Figure 1 depicts average RBV curves grouped by six different FO levels between 0 and 5 liters (table 3). Only treatments with a treatment time $>245$ min (corresponding to the 10th percentile of treatment times) and UFR in

\section{Subgroup Analysis}

Subgroup analysis was performed including only treatments within the interquartile UFR range (25th to 
Table 3. Data overview to figure 1 (RBV curves at different FO levels)

\begin{tabular}{|c|c|c|c|c|c|c|}
\hline & \multicolumn{6}{|l|}{ FO group, 1} \\
\hline & -0.5 to 0.5 & 0.5 to 1.5 & 1.5 to 2.5 & 2.5 to 3.5 & 3.5 to 4.5 & 4.5 to 5.5 \\
\hline Patients, $\mathrm{n}$ & 7 & 11 & 15 & 16 & 7 & 4 \\
\hline Treatments, $\mathrm{n}$ & 23 & 32 & 35 & 31 & 17 & 10 \\
\hline UFV, 1 & $2.7 \pm 0.5$ & $2.6 \pm 0.4$ & $2.6 \pm 0.4$ & $2.7 \pm 0.3$ & $2.9 \pm 0.3$ & $2.6 \pm 0.3$ \\
\hline $\mathrm{UFR}, \mathrm{ml} / \mathrm{h}$ & $545 \pm 82$ & $532 \pm 79$ & $538 \pm 77$ & $555 \pm 69$ & $567 \pm 61$ & $511 \pm 57$ \\
\hline UFR index, $\mathrm{ml} / \mathrm{h} / \mathrm{kg}$ & $8.2 \pm 2.0$ & $7.8 \pm 1.8$ & $8.2 \pm 2.1$ & $9.3 \pm 2.5$ & $8.1 \pm 1.4$ & $6.9 \pm 1.1$ \\
\hline SBP before HD, mm Hg & $113 \pm 25$ & $124 \pm 17$ & $131 \pm 33$ & $140 \pm 27$ & $140 \pm 20$ & $153 \pm 15$ \\
\hline DBP before $\mathrm{HD}, \mathrm{mm} \mathrm{Hg}$ & $61 \pm 18$ & $61 \pm 12$ & $56 \pm 15$ & $66 \pm 17$ & $64 \pm 18$ & $62 \pm 6$ \\
\hline RBV slope full treatment, $\% / h$ & -2.1 & -1.6 & -1.0 & -1.2 & -0.6 & -0.3 \\
\hline RBV slope first $30 \mathrm{~min}, \% / \mathrm{h}$ & -6.0 & -6.0 & -7.6 & -4.8 & -6.0 & -1.2 \\
\hline RBV slope last $30 \mathrm{~min}, \% / \mathrm{h}$ & -3.8 & -2.5 & -2.2 & -2.2 & -0.8 & -1.2 \\
\hline $\mathrm{RBV} \%$ at $260 \mathrm{~min}, \%$ & $88.0 \pm 4.8$ & $90.4 \pm 3.9$ & $91.0 \pm 4.7$ & $92.5 \pm 4.9$ & $94.0 \pm 3.2$ & $97.7 \pm 5.4$ \\
\hline Slope 4 h, \%/h/l/h & $-3.9 \pm 0.6$ & $-3.0 \pm 0.4$ & $-1.9 \pm 0.4$ & $-2.2 \pm 0.3$ & $-1.0 \pm 0.1$ & $-0.6 \pm 0.1$ \\
\hline Volume index, $\% / \mathrm{h} / \mathrm{ml} / \mathrm{h} / \mathrm{kg}$ & $-0.27 \pm 0.06$ & $-0.22 \pm 0.07$ & $-0.14 \pm 0.04$ & $-0.14 \pm 0.04$ & $-0.08 \pm 0.01$ & $-0.05 \pm 0.01$ \\
\hline
\end{tabular}

Only treatments with a UF rate between the 25 th and 75 th percentile $(400-675 \mathrm{ml} / \mathrm{h})$ and treatment time $>10$ th percentile $(>245$ min) were included.

the interquartile range as described above were included $(\mathrm{n}=148)$. Table 3 shows that average UFVs and UFRs were comparable in all six FO groups. Every patient contributed no more than once to each of the six curves in the diagram. If a patient had more than one RBV curve in one of the FO groups which was the case in most patients, then these RBV curves were averaged individually before being assigned to the respective group average. This procedure ensured that all patients had the same relative contribution to the average RBV curves. The number of treatments that each patient contributed is summarized in table 3. RBV curves were steep for low FO and became flatter with increasing FO. Predialysis SBP steadily increased with FO (from $113 \mathrm{~mm} \mathrm{Hg}$ in the 0-liter group to $153 \mathrm{~mm} \mathrm{Hg}$ in the 5-liter group).

\section{Volume Marker Sensitivity}

Figure 2 shows ROC curves for three different FO cutoff values (2, 3, and 4 liters), using the Slope $4 \mathrm{~h}$ marker as the continuous variable $(\mathrm{n}=317)$. The AUC to predict FO was $0.63,0.69$, and 0.84 for $\mathrm{FO}>2,3$, and 4 liters, respectively. The best discriminatory power was therefore achieved for $\mathrm{FO}>4$ liters.

Figure 3 shows the AUCs for the Slope $4 \mathrm{~h}$ marker together with the corresponding 95\% CI for all data. The AUC curve had the shape of a bathtub, with lowest values between 1.5 and 2.5 liters, and increasing to both lower and higher FO levels. The highest AUC values were achieved at FO levels $>4$ liters, indicating better perfor- mance of the Slope4h marker in detecting high FO. The subgroup analysis revealed even higher AUC levels (up to 0.90 ) when compared with the complete dataset.

\section{Correlations between Volume Markers and Fluid \\ Overload}

The correlations between the different volume markers and FO are listed in table 1. The strongest correlation was found in Slope4h, both in all data and the subgroup analysis $(\mathrm{R}=0.33$ and 0.52 , respectively). The correlation between SBP and Slope $4 \mathrm{~h}$ was $\mathrm{R}=0.14(\mathrm{p}=0.02)$ for all data, and $R=0.27(p=0.001)$ in the subgroup analysis.

The average RBV drop was slightly lower in diabetic patients than in non-diabetics $\left(\mathrm{RBV}_{\text {end }}=90\right.$ vs. $88 \%$, respectively, $\mathrm{p}=0.048)$. FO was about 1 liter higher in diabetic patients.

\section{Discussion}

BVM is used mostly to assess intradialytic vascular depletion due to extensive ultrafiltration in order to avoid hypotensive episodes. This work concentrates on a different aspect - the use of BVM for fluid status assessment.

We found that (i) BV monitoring appears well suited for an overall qualitative fluid status assessment in patient 


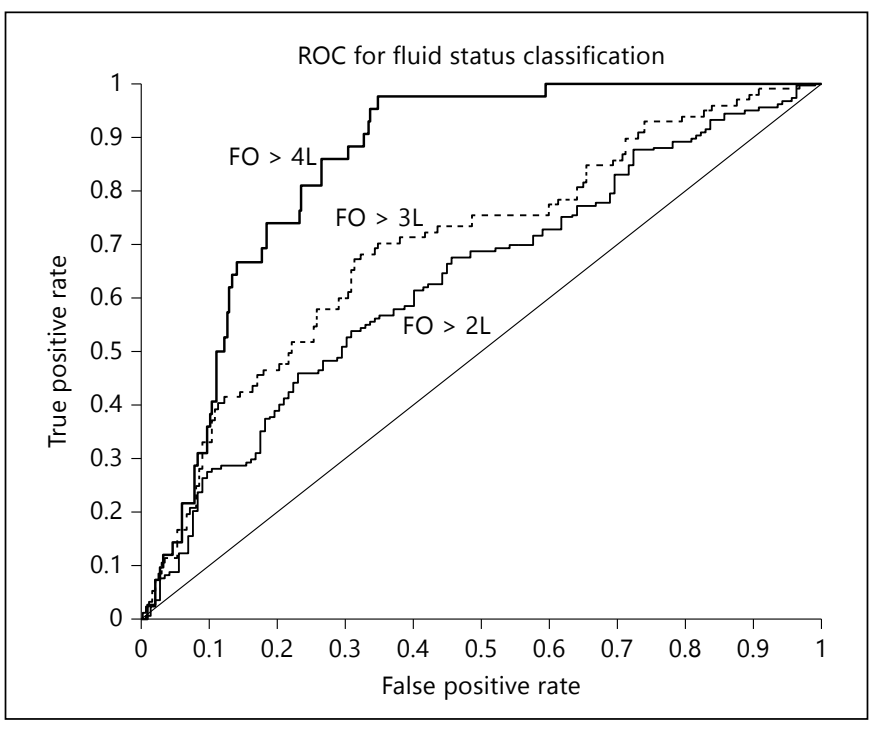

Fig. 2. ROC curves for three different FO cutoff values (2, 3, and 4 liters), using the Slope $4 \mathrm{~h}$ marker as the continuous variable.

groups, but suffers from a high variability on the individual level, and (ii) the sensitivity of BVM markers over a wide FO range has the shape of a bathtub, being moderate in middle $\mathrm{FO}$ ranges between about 1 and 3 liters, slightly better in low fluid status $<1$ liter, and best in detecting high $\mathrm{FO}>3-4$ liters.

\section{Relationship between Blood Volume Changes and Fluid Overload}

BVM assesses the balance between refilling and UFR in respect to the absolute blood volume. FO is one of many factors influencing this relationship. It has often been reported that flat RBV curves are a sign of excessive FO $[6,8,9,11]$. According to Guyton and Hall [24], about two thirds of FO contribute to the interstitial space, and the remaining third contributes to the blood volume. Both effects result in a flatter RBV curve: firstly, interstitial FO provides a fluid reservoir that facilitates refilling from the interstitial to the vascular space and secondly, a 1 -liter decrease in blood volume will lead to a smaller relative change if the absolute blood volume is higher.

The correlation that we found between Slope $4 \mathrm{~h}$ and FO $\left(\mathrm{R}^{2}=0.11\right)$ indicates that only $11 \%$ of the variability of the BVM marker can be explained by FO. The explained variability increases to $27 \%$ in a subgroup analysis where we used only treatments with comparable UFRs. The remaining variability is likely to be caused by non-FO-related effects like individual differences in absolute blood volume, albumin levels, fluid shifts induced

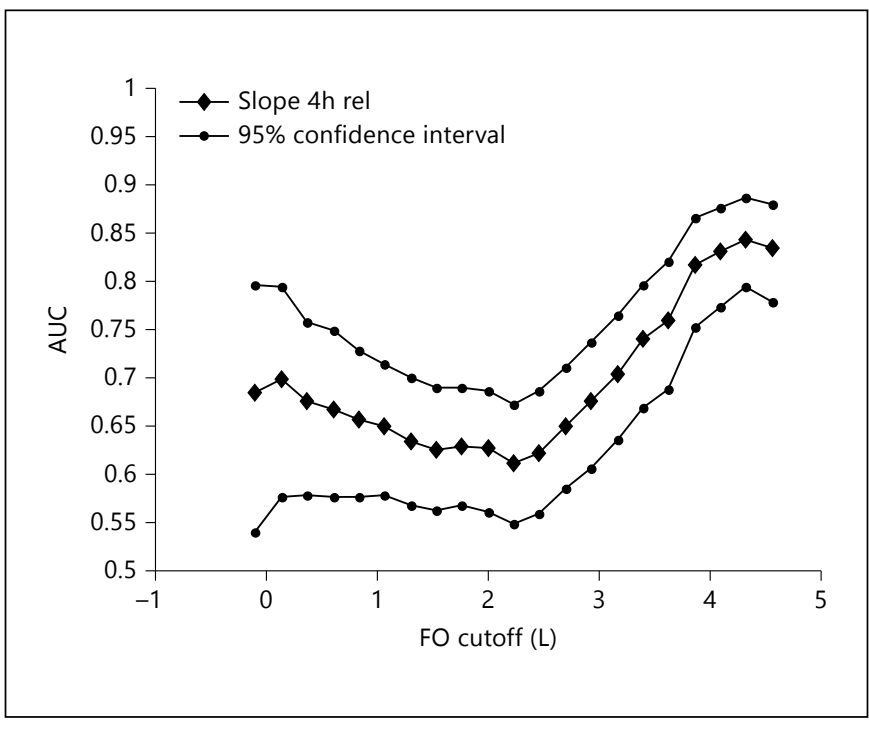

Fig. 3. AUC and $95 \%$ CI for ROC classifications of FO using the Slope 4 h marker.

by postural changes or the mixing of blood from different vascular beds and different hematocrit levels (Fåhræus-Lindqvist effect). It is obvious that these limitations are independent of the measurement technique used by different manufacturers (e.g. optical or ultrasound based), and that the results apply to all devices measuring on the extracorporeal blood line. Other markers like the slopes of the first and last $30 \mathrm{~min}$ of the treatments were not as well correlated with FO as the slope over the complete treatment. Predialysis SBP was a good marker of FO in all data, but did not further improve in the subgroup analysis. The finding that $\mathrm{BP}$ correlated well with FO raises questions about the effectiveness of antihypertensive medication. Normalizing FO may be an important alternative to antihypertensive drugs in a significant number of patients. Even though the individual variability is high, figure 1 demonstrates that average RBV curves of many treatments and patients are well linked to predialysis FO. The lower the FO, the more pronounced was the RBV curve decrease. Interestingly, the groups 1-4 liters are relatively close together, while 0 and 5 liters have a greater distance to the other curves. It also appears that the 0 -liter group, after almost plateauing at $150 \mathrm{~min}$, continues to drop steeply towards the end of the treatment, indicating a more rapid depletion of blood volume as compared to the other groups. Mitra et al. [25] have related the transition from an RBV plateau into an RBV drop to depletion of interstitial fluid space when dry weight has been reached. In this situ- 
ation, refilling is no longer sufficient to maintain constant blood volume.

The fact that the 5-liter curve is well separated from the other curves indicates that a flat curve is indeed a rather reliable indicator of excessive FO. Dasselaar et al. [26] noted that RBV curves normalized by UFR were flatter during the first session of the week as compared to the second and third sessions, which is consistent with our results assuming FO levels are higher after the long interdialytic interval. FO levels were also higher in the subgroup of diabetic patients, which is probably the reason why they presented slightly less average RBV drop than non-diabetics, even though their average UFR was about $15 \%$ higher.

\section{Sensitivity of BVM Markers for Fluid Status \\ Assessment}

In order to assess the sensitivity of BVM markers for identifying different FO levels, we performed a ROC analysis for three different FO cutoff levels (2, 3, and 4 liters), as shown in figure 2. The largest AUC is reached for the 4-liter cutoff, demonstrating that the Slope4h marker is well suited to discriminate between FO levels $>4$ and $<4$ liters, irrespective of UFR. The ROC curve for the 2-liter cutoff is much lower, indicating less performance for the assessment of moderate FO. Figure 3 provides a more detailed insight into the sensitivity over a wide FO range. The sensitivity rises strongly for FO levels $>3$ liters, a result which is in accordance with the findings of Agarwal et al. [16] who were able to detect excess fluid but not depletion when compared against echocardiographic markers of volume status. The Slope $4 \mathrm{~h}$ sensitivity in figure 3 increases also at low FO levels, but not as strongly as compared to excess volume. One may speculate that the sensitivity would further increase at more negative predialysis FO levels, but in our study -0.1 liter represented the lower 10th percentile of predialysis FO, thus not enough data was available to prove this hypothesis.

\section{Limitations}

Our patients had a relatively constant intraindividual predialysis FO over the seven treatments, therefore we could not demonstrate the effect of within-patient differences in predialysis fluid status on RBV slopes. Furthermore, we performed only one real FO measurement per patient. Additional FO values were calculated from preweight differences which may have added additional variability to the results arising for example from clothing differences. This would potentially underestimate the true correlation between FO and PV markers, but not change the general finding of different sensitivity ranges. Finally, given the retrospective nature of our investigation, it is not possible to extrapolate these findings to the general dialysis population. Further studies are needed which isolate the effect of FO on PV reduction by prospectively controlling factors that influence refilling (e.g. UFR or albumin). These studies should also compare different predialysis FO levels in the same patient, e.g. by measuring treatments after the long and short interdialytic interval.

\section{Conclusion}

BVM may assist fluid management in hemodialysis patients by identifying states of volume excess or depletion, but its utility for fine tuning at normal or moderate FO levels is questionable.

\section{References}

$>1$ Sinha AD, Agarwal R: Can chronic volume overload be recognized and prevented in hemodialysis patients? The pitfalls of the clinical examination in assessing volume status. Semin Dial 2009;22:480-482.

-2 Arneson TJ, Liu J, Qiu Y, Gilbertson DT, Foley RN, Collins AJ: Hospital treatment for fluid overload in the Medicare hemodialysis population. Clin J Am Soc Nephrol 2010;5:1054-1063.

$>3$ Wizemann V, Wabel P, Chamney P, et al: The mortality risk of overhydration in haemodialysis patients. Nephrol Dial Transplant 2009; 24:1574-1579.
4 Wabel P, Moissl U, Chamney P, et al: Towards improved cardiovascular management: the necessity of combining blood pressure and fluid overload. Nephrol Dial Transplant 2008; 23:2965-2971.

5 Machek P, Jirka T, Moissl U, Chamney P, Wabel P: Guided optimization of fluid status in haemodialysis patients. Nephrol Dial Transplant 2010;25:538-544.

$>6$ Wizemann V, Leibinger A, Mueller K, Nilson A: Influence of hydration state on plasma volume changes during ultrafiltration. Artif Organs 1995;19:416-419.

7 Charra B: Fluid balance, dry weight, and blood pressure in dialysis. Hemodial Int 2007; 11:21-31.
$>8$ Agarwal R, Weir MR: Dry weight: a concept revisited in an effort to avoid medication-directed approaches for blood pressure control in hemodialysis patients. Clin J Am Soc Nephrol 2010;5:1255-1260.

$\checkmark 9$ Dasselaar JJ, van der Sande FM, Franssen CF: Critical evaluation of blood volume measurements during hemodialysis. Blood Purif 2012; 33:177-182.

10 Lopot F, Nyiomnaitham V, Svarova, Polakovic V, Svara F, Sulkova S: Continuous blood volume monitoring and 'dry weight' assessment. J Ren Care 2007;33:52-58. 
11 Sinha AD, Light RP, Agarwal R: Relative plasma volume monitoring during hemodialysis aids the assessment of dry weight. Hypertension 2010;55:305-311.

$\checkmark 12$ Rodriguez HJ, Domenici R, Diroll A, Goykhman I: Assessment of dry weight by monitoring changes in blood volume during hemodialysis using Crit-Line. Kidney Int 2005;68:854-861.

13 Bonello M, House AA, Cruz D, et al: Integration of blood volume, blood pressure, heart rate and bioimpedance monitoring for the achievement of optimal dry body weight during chronic hemodialysis. Int J Artif Organs 2007;30:1098-1108.

$\checkmark 14$ Lopot F, Kotyk P, Blaha J, Forejt J: Use of continuous blood volume monitoring to detect inadequately high dry weight. Int J Artif Organs 1996;19:411-414.

15 Yu SJ, Kim DH, Oh DJ, Yu SH, Kang ET: Assessment of fluid shifts of body compartments using both bioimpedance analysis and blood volume monitoring. J Korean Med Sci 2006; 21:75-80.
16 Agarwal R, Kelley K, Light RP: Diagnostic utility of blood volume monitoring in hemodialysis patients. Am J Kidney Dis 2008;51:242-254.

17 Dasselaar JJ, de Jong PE, Huisman RM, Franssen CF: Effect of high and low ultrafiltration volume during hemodialysis on relative blood volume. ASAIO J 2006;52:169-173.

18 Krepel HP, Nette RW, Akcahuseyin E, Weimar W, Zietse R: Variability of relative blood volume during haemodialysis. Nephrol Dial Transplant 2000;15:673-679.

19 Cole KS, Cole RH: Dispersion and absorption in dielectrics. 1. Alternating current characteristics. J Chem Phys 1941;9:341-351.

20 Chamney PW, Wabel P, Moissl UM, et al: A whole-body model to distinguish excess fluid from the hydration of major body tissues. Am J Clin Nutr 2007;85:80-89.
21 Moissl UM, Wabel P, Chamney PW, et al: Body fluid volume determination via body composition spectroscopy in health and disease. Physiol Meas 2006;27:921-933.

22 Fuller NJ, Jebb SA, Laskey MA, Coward WA, Elia M: Four-component model for the assessment of body composition in humans: comparison with alternative methods, and evaluation of the density and hydration of fatfree mass. Clin Sci (Lond) 1992;82:687-693.

23 Wabel P, Chamney P, Moissl U, Jirka T: Importance of whole-body bioimpedance spectroscopy for the management of fluid balance. Blood Purif 2009;27:75-80.

24 Guyton AC, Hall JE: Textbook of Medical Physiology. Philadelphia,Saunders, 2000.

25 Mitra S, Chamney P, Greenwood R, Farrington $\mathrm{K}$ : Linear decay of relative blood volume during ultrafiltration predicts hemodynamic instability. Am J Kidney Dis 2002;40:556-565.

-26 Dasselaar JJ, de Jong PE, Huisman RM, Franssen CF: Influence of ultrafiltration volume on blood volume changes during hemodialysis as observed in day-of-the-week analysis of hemodialysis sessions. ASAIO J 2007;53:479-484. 